\title{
The telegraph equation for cosmic-ray transport with weak adiabatic focusing ${ }^{\star}$
}

\author{
Y. E. Litvinenko ${ }^{1,2}$ and R. Schlickeiser ${ }^{1}$ \\ 1 Institut für Theoretische Physik, Lehrstuhl IV: Weltraum- und Astrophysik, Ruhr-Universität Bochum, 44780 Bochum, Germany \\ 2 Department of Mathematics, University of Waikato, PB 3105, Hamilton, New Zealand
}

Received 20 February 2013 / Accepted 2 May 2013

\begin{abstract}
Time-dependent solutions of a spatial diffusion equation are often used to describe the transport of solar energetic particles, accelerated in large solar flares. Approximate analytical solutions of the diffusion approximation can complement and guide detailed numerical solutions of the Fokker-Planck equation for the particle distribution function. The accuracy of the diffusion approximation is limited, however, because the signal propagation speed is infinite in the diffusion limit. An improved description of cosmic-ray transport is provided by the telegraph equation, characterised by a finite signal propagation speed. We derive the telegraph equation for the particle density, taking into account adiabatic focusing in a large-scale interplanetary magnetic field in a weak focusing limit. As an illustration, we calculate a propagating pulse solution of the telegraph equation, determine the rise time when the maximum particle intensity is reached at a given distance from the Sun, and compare the results with those obtained in the diffusion approximation. In comparison with the diffusion equation, the telegraph equation predicts an asymmetrical shape of the pulse and a shorter rise time. These potentially significant differences suggest that the more accurate telegraph equation should be used in analysis of the solar energetic particle data, at least to quantify the accuracy of the focused diffusion model.
\end{abstract}

Key words. Sun: particle emission - scattering - magnetic fields - diffusion - cosmic rays

\section{Introduction}

The transport of energetic particles in turbulent cosmic magnetic fields is typically described using the Fokker-Planck equation for the particle distribution function (for a recent derivation, see Schlickeiser 2011). The diffusion approximation for the particle density is a well-known simplified form of the Fokker-Planck equation, which results when turbulent pitch-angle scattering is strong enough to ensure that the scale of density variation is significantly greater than the particle mean free path (Jokipii 1966; Hasselmann \& Wibberenz 1968, 1970; Earl 1974). Numerical studies confirmed the accuracy of the diffusion approximation in a uniform mean magnetic field (Kóta et al. 1982).

Large-scale magnetic fields, however, are often non-uniform in space plasmas. Roelof (1969), Earl (1976), and Kunstmann (1979) identified the effect of adiabatic focusing on particle transport in spatially varying magnetic fields. Focused transport equations have been used extensively to model the propagation of energetic particles in interplanetary space following large solar flares (e.g., Bieber et al. 2002; Sáiz et al. 2008; Dröge et al. 2010).

Adiabatic focusing modifies the coefficient of particle diffusion parallel to the mean magnetic field and causes coherent particle streaming along the field. Earl (1981) and Beeck \& Wibberenz (1986) obtained the diffusion equation taking adiabatic focusing into account (for a recent discussion, see Litvinenko 2012). Schlickeiser \& Shalchi (2008) gave a systematic derivation of the diffusion approximation in a weak focusing limit, when the magnetic focusing length is much greater

\footnotetext{
* Appendix $\mathrm{A}$ is available in electronic form at http://www. aanda.org
}

than the scattering length of cosmic-ray particles. In this limit, the main effect of a non-uniform mean magnetic field is the convection-like coherent streaming, whereas the parallel diffusion coefficient is independent of the focusing length.

Litvinenko \& Noble (2013) used numerical simulations to emphasise the limitations of the diffusion approximation to focused particle transport, related to the fact that the propagation speed is infinite in the diffusion limit. Despite it limitations, the diffusion approximation is often used to model focused particle transport in astrophysics (e.g., Artmann et al. 2011; Tautz et al. 2012, and references therein).

An improved description of cosmic-ray transport is provided by the telegraph equation, characterised by a finite signal propagation speed, which is obtained from an eigenfunction expansion of the distribution function. Alternative derivations of the telegraph equation are available in the limit of a uniform magnetic field (Earl 1974; Gombosi et al. 1993; for a detailed discussion of the alternative derivations, see Schwadron \& Gombosi 1994). In the case of focused transport, however, the complication arises that the telegraph equation describes the coefficient of an eigenfunction expansion rather than the particle density that is the physical quantity of interest (Earl 1976). Litvinenko \& Noble (2013) used a new technique to obtain the telegraph equation for the particle density in the case of an arbitrary focusing strength, but their derivation is only valid under the assumption of isotropic pitch-angle scattering.

The purpose of this paper is to generalise the calculations of Earl (1974, 1976) and Beeck \& Wibberenz (1986) and derive the telegraph equation for the particle density in the weak focusing limit of Schlickeiser \& Shalchi (2008). The resulting equation is valid for an anisotropic scattering rate, complementing 
the derivation given by Litvinenko \& Noble (2013). We also use the new transport equation to re-examine the propagation of energetic particles produced in solar flares. As an illustration, we compare the temporal profiles, predicted by the fundamental solution to the telegraph equation, with those calculated by Schlickeiser \& Shalchi (2008) in the diffusion approximation. Comparison of the predicted profiles allows us to quantify the accuracy of the diffusion approximation in a concrete application.

\section{The Fokker-Planck equation and the diffusion approximation}

The Fokker-Planck equation for a cosmic-ray distribution function, which incorporates the effects of pitch-angle scattering and adiabatic focusing, is given by

$\frac{\partial f}{\partial t}+\mu v \frac{\partial f}{\partial z}+\frac{v}{2 L}\left(1-\mu^{2}\right) \frac{\partial f}{\partial \mu}=\frac{\partial}{\partial \mu}\left(D_{\mu \mu} \frac{\partial f}{\partial \mu}\right)$

(e.g., Roelof 1969; Earl 1981). Here $f$ is the distribution function of energetic particles (gyrotropic phase-space density), $t$ is time, $\mu$ is the cosine of the particle pitch angle, $v$ is the particle speed, $z$ is the distance along the mean magnetic field $\boldsymbol{B}_{0}=B_{0} \hat{z}$, $L=-B_{0} /\left(\partial B_{0} / \partial z\right)$ is the adiabatic focusing length, and $D_{\mu \mu}$ is the Fokker-Planck coefficient for pitch-angle scattering. Note for clarity that Litvinenko \& Noble (2013) used $f_{0}$ to denote the phase-space density and $f$ to denote the linear density (Earl 1981). We use a simpler notation since we do not work with the linear density.

The following assumptions, adopted throughout this paper, simplify the analytical treatment. Momentum diffusion is neglected, which is typically justified for the transport of solar energetic particles (SEPs) in interplanetary space (e.g., Artmann et al. 2011). A constant focusing length $L$ is adopted, which is not a significant limitation as long as $L$ does not change much within one scattering length $\lambda$. The condition $\lambda \ll L$ is in fact the weak focusing limit of Schlickeiser \& Shalchi (2008), which yields $\lambda \approx \lambda_{0}$ where $\lambda_{0}$ is the scattering length in the absence of focusing. Note, however, that the focusing length in the Parker spiral of magnetic field is not constant, and $\lambda / L$ is not small sufficiently close to the sun. In any case, a diffusive transport model is not accurate for strongly anisotropic particle distributions close to the sun. Finally, the only restriction placed on the scattering rate is that $D_{\mu \mu}$ is an even function of $\mu$, so that, for instance, backward- and forward-propagating waves should have equal intensities in the slab turbulence model. Since generally $D_{\mu \mu} \sim\left(1-\mu^{2}\right)$ (Jokipii 1966), the scattering coefficient vanishes at $\mu= \pm 1$.

We begin by briefly reviewing the derivation of the diffusion approximation. Following Beeck \& Wibberenz (1986), we write the distribution function as the sum of the isotropic density $F$ and an anisotropic component $g$ :

$f(z, \mu, t)=F(z, t)+g(z, \mu, t)$,

where

$\int_{-1}^{1} g \mathrm{~d} \mu=0$

Integration of Eq. (1) over $\mu$ gives

$\frac{\partial F}{\partial t}+\left(\frac{\partial}{\partial z}+\frac{1}{L}\right) S=0$ where

$S=\frac{v}{2} \int_{-1}^{1} \mu g \mathrm{~d} \mu=\frac{v}{4} \int_{-1}^{1}\left(1-\mu^{2}\right) \frac{\partial g}{\partial \mu} \mathrm{d} \mu$.

To calculate the flux $S$, we rewrite Eq. (1) as follows:

$\left(\frac{\partial F}{\partial t}+\frac{\partial g}{\partial t}+\mu v \frac{\partial g}{\partial z}\right)+\mu v \frac{\partial F}{\partial z}=\frac{\partial}{\partial \mu}\left(D_{\mu \mu} \frac{\partial g}{\partial \mu}\right)$

where a term proportional to $1 / L$ is neglected in the weak focusing limit.

The diffusion approximation is obtained by neglecting the bracketed terms on the left-hand side of Eq. (6). Then Eq. (6) can be integrated twice, and the two integration constants are specified using the condition $D_{\mu \mu}( \pm 1)=0$ and Eq. (3). The resulting expression for the anisotropic part of the distribution function is as follows:

$g=-\frac{v}{2} \frac{\partial F}{\partial z} \int_{0}^{\mu} \frac{1-\mu^{\prime 2}}{D_{\mu \mu}\left(\mu^{\prime}\right)} \mathrm{d} \mu^{\prime}$.

On substituting this expression in Eq. (5), we get

$S=-\kappa_{\|} \frac{\partial F}{\partial z}$

where it is customary to express the parallel diffusion coefficient

$\kappa_{\|}=\frac{1}{3} \lambda_{0} v$

in terms of the scattering length

$\lambda_{0}=\frac{3 v}{8} \int_{-1}^{1} \frac{\left(1-\mu^{2}\right)^{2}}{D_{\mu \mu}(\mu)} \mathrm{d} \mu$.

Using this expression for the flux in Eq. (4) yields the diffusion approximation for the particle density in the weak focusing limit:

$\frac{\partial F}{\partial t}=\kappa_{\|} \frac{\partial^{2} F}{\partial z^{2}}+\frac{\kappa_{\|}}{L} \frac{\partial F}{\partial z}$

The main consequence of adiabatic focusing is the appearance of a convective term in the diffusion model, characterised by the coherent speed $u=\kappa_{\|} / L$. Because this mode of particle transport comprises both coherent streaming and diffusion, Earl (1981) termed it pseudo-diffusion.

\section{The telegraph equation for focused transport}

The treatment so far basically follows the standard arguments leading to the diffusion approximation for cosmic-ray transport (Beeck \& Wibberenz 1986; Schlickeiser \& Shalchi 2008). To obtain an improved description of the particle transport, we use an iterative method to solve Eq. (6) and determine the anisotropic part $g$ of the distribution function more accurately.

We substitute the diffusion-limit Eq. (7) into the left-hand side of Eq. (6), determine $\partial g / \partial \mu$ by integrating with respect to $\mu$, and use Eq. (5) to calculate the flux $S$. Note that, while calculating second-iteration terms in the weak focusing limit, we can replace $\kappa_{\|} \partial_{z z} F$ by $\partial_{t} F$ since the difference would only give rise to terms proportional to higher powers of the small focusing parameter $\lambda_{0} / L$ which we neglect. It is an exercise in first-year calculus to show, using integration by parts, that the contributions of the 
first and third terms on the left-hand side of Eq. (6) to the flux $S$ cancel each other. The remaining terms can be rewritten as

$S=-\kappa_{\|} \frac{\partial F}{\partial z}+\frac{v}{4} \int_{-1}^{1}\left(\int_{-1}^{\mu} \frac{\partial g}{\partial t} \mathrm{~d} \mu^{\prime}\right) \frac{\left(1-\mu^{2}\right)}{D_{\mu \mu}(\mu)} \mathrm{d} \mu$

which, on integrating by parts and substituting Eq. (7), yields

$S=-\kappa_{\|} \frac{\partial F}{\partial z}+\tau \kappa_{\|} \frac{\partial^{2} F}{\partial z \partial t}$,

where

$\tau=\int_{-1}^{1}\left[\int_{0}^{\mu} \frac{1-\mu^{\prime 2}}{D_{\mu \mu}\left(\mu^{\prime}\right)} \mathrm{d} \mu^{\prime}\right]^{2} \mathrm{~d} \mu / \int_{-1}^{1} \frac{\left(1-\mu^{2}\right)^{2}}{D_{\mu \mu}(\mu)} \mathrm{d} \mu$

Next we substitute the expression for $S$ into Eq. (4) and use Eq. (11) to simplify the terms proportional to $\tau$, which only introduces an error term proportional to third derivatives of $F$ that we neglect. The resulting equation for $F(z, t)$ in the weak focusing limit is a generalised telegraph equation:

$\frac{\partial F}{\partial t}+\tau \frac{\partial^{2} F}{\partial t^{2}}=\kappa_{\|} \frac{\partial^{2} F}{\partial z^{2}}+\frac{\kappa_{\|}}{L} \frac{\partial F}{\partial z}$

Although the telegraph equation formally reduces to the diffusion model when $\tau=0$, in practice it cannot be assumed that $\tau$ is a negligibly small parameter. Suppose, for instance, that the random magnetic fields superposed on the mean field $B_{0}$ are characterised by a power-law spectrum of the magnetic energy per unit wave-number interval in fluctuations perpendicular to $B_{0}$. Then the quasilinear approximation (Jokipii 1966) leads to the pitch-angle scattering rate

$D_{\mu \mu}=D_{0}|\mu|^{q-1}\left(1-\mu^{2}\right)$,

where $D_{0}=$ const and $1 \leq q<2$ is the spectral index of the power law. Evaluation of the integrals in Eq. (14) yields

$D_{0} \tau=\frac{1}{2} \frac{(4-q)}{(5-2 q)(2-q)}$.

Thus $D_{0} \tau=1 / 2$ for isotropic scattering $(q=1)$, and $\tau$ is an increasing function of $q$ in the range of applicability of the quasilinear theory. Alternatively,

$\tau=\frac{\lambda_{0}}{v} \frac{(4-q)^{2}}{3(5-2 q)}$

where Eq. (10) was used to calculate the scattering length $\lambda_{0}$.

It is convenient to introduce dimensionless variables, by measuring distances in units of the scattering length $\lambda_{0}$, speeds in units of the constant particle speed $v$, and times in units of $\lambda_{0} / v$. The parallel diffusion coefficient, normalised by $\lambda_{0} v$, is $\kappa_{\|}=1 / 3$, and Eq. (15) in dimensionless form is as follows:

$$
\frac{\partial F}{\partial t}+\tau \frac{\partial^{2} F}{\partial t^{2}}=\frac{1}{3} \frac{\partial^{2} F}{\partial z^{2}}+\frac{\xi}{3} \frac{\partial F}{\partial z}
$$

where $\xi=\lambda_{0} / L$ is a dimensionless focusing parameter. It is worth stressing that the dimensionless parameter $1 \leq \tau<4 / 3$ is not small for any value of $q$ in the range $1 \leq q<2$.

So far we worked in the rest frame of a plasma. When cosmic-ray particles propagate in a moving plasma, say in the solar wind, it is useful to describe the particle transport in the laboratory (observer) reference frame. In the Appendix we examine the case of a plasma moving with respect to the observer with a bulk speed $U$ along the mean magnetic field. The result of a fully relativistic analysis is a modified telegraph equation. However, if the plasma flow is non-relativistic, $U / c \ll 1$, and adiabatic energy changes due to a gradient of $U$ are neglected, then the only modification of the transport equation is given by a convective term $U \partial_{z} F$ that can be easily incorporated into the dimensionless Eq. (19) by generalising the definition of the focusing parameter:

$\xi=\frac{\lambda_{0}}{L}-3 \frac{U}{v}$.

Below we use the telegraph equation for focused particle transport to illustrate how the accuracy of the diffusion approximation can be quantified in a specific application.

\section{An application to the transport of solar energetic particles}

Solar energetic particles, accelerated in large solar flares, propagate in interplanetary space and interact with a turbulent interplanetary magnetic field. Early observations suggested that the resulting particle transport is diffusive (Meyer et al. 1956; Roelof 1969). Hence a simple description of the SEP transport can be provided by time-dependent solutions of a spatial diffusion equation with a diffusion coefficient determined by the spectrum of the turbulence (e.g., Kóta et al. 1982; Artmann et al. 2011, and references therein). Note for clarity that some SEP distributions are strongly anisotropic, in which case the particle transport is not diffusive but rather effectively scatter-free (e.g., Haggerty \& Roelof 2002).

Schlickeiser \& Shalchi (2008) considered how coherent streaming due to adiabatic focusing in a large-scale interplanetary magnetic field modifies the diffusive SEP transport. Specifically Schlickeiser \& Shalchi (2008) calculated how adiabatic focusing modifies the shape of a propagating density pulse and used the results to suggest an alternative interpretation of the observed solar scatter-free events. The argument is based on a solution to a spatial diffusion equation for the particle density, which incorporates the effects of adiabatic focusing. In our present notation, the diffusion equation used by Schlickeiser \& Shalchi (2008) is Eq. (19) with $\tau=0$, and a propagating SEP pulse is modelled by the fundamental solution

$F(z, t)=\frac{1}{(4 \pi t / 3)^{1 / 2}} \exp \left[-\frac{(z+\xi t / 3)^{2}}{4 t / 3}\right]$.

A quantity of practical interest is the SEP rise time, defined as the time when the maximum particle intensity is reached at a given distance $z$ from the Sun. The time $t_{\mathrm{m}}$ for $F(z, t)$ to reach a maximum is determined by solving $\mathrm{d} F / \mathrm{d} t=0$, which yields

$t_{\mathrm{m}}=\frac{3}{\xi^{2}}\left(\sqrt{1+\xi^{2} z^{2}}-1\right)$.

While the standard diffusion result $t_{\mathrm{m}}=3 z^{2} / 2$ is recovered in the limit $\xi \rightarrow 0$, a qualitatively new behaviour $t_{\mathrm{m}} \approx 3 z /|\xi|$ is predicted in the range $|\xi| z>1$. The key point is that, regardless of the sign of $\xi$, adiabatic focusing effects dominate over diffusion, leading to seemingly non-diffusive temporal profiles with $t_{\mathrm{m}} \sim z$ in SEP events.

Another useful quantity, predicted in the diffusion approximation, is the streaming anisotropy of the particle distribution, defined as

$A(z, t)=\frac{3 \int_{-1}^{1} \mu f \mathrm{~d} \mu}{\int_{-1}^{1} f \mathrm{~d} \mu}=\frac{3 S}{v F}$. 
Equation (8) for the diffusive flux $S$ gives

$A(z, t)=-\frac{1}{F} \frac{\partial F}{\partial z}$

in dimensionless variables, which yields, on substituting the fundamental solution given by Eq. (21),

$A(z, t)=\frac{\xi}{2}+\frac{3 z}{2 t}$.

The formula gives a reasonably accurate fit to the observed SEP anisotropy profiles (Schlickeiser et al. 2009; Artmann et al. 2011). However, since $|A| \leq 3$ by definition, an unsatisfactory feature, related to the infinite signal propagation speed of the diffusion equation, is that $A \rightarrow \infty$ as $t \rightarrow 0$.

As a concrete illustration of how the telegraph equation for focused particle transport could be used in practice, we now calculate the shape of a travelling SEP pulse and its dependence on the strength of adiabatic focusing in the more accurate model of Eq. (15) and compare the results with those obtained in the diffusion approximation. The fundamental solution of Eq. (19) on the infinite interval $-\infty<z<\infty$ (with the source term $\delta(z) \delta(t)$ on the right-hand side) is given by

$F(z, t)=\sqrt{\frac{3}{4 \tau}} \exp \left(-\frac{\xi z}{2}-\frac{t}{2 \tau}\right) I_{0}(s)$

for $|z|<t / \sqrt{3 \tau}$, and zero otherwise (e.g., Kevorkian 2000). Here $I_{0}$ is a modified Bessel function, and

$s=\frac{1}{2} \sqrt{\left(1-\frac{\xi^{2} \tau}{3}\right)\left(\frac{t^{2}}{\tau^{2}}-\frac{3 z^{2}}{\tau}\right)}$.

Equation (26) formally reduces to Eq. (21) in the limit $\tau \rightarrow 0$.

The travelling pulse solution given by Eq. (26) is valid as long as

$\frac{1}{3} \xi^{2} \tau<1$

This condition should be satisfied in the weak focusing limit. For example, Schlickeiser et al. (2009) estimated $\xi \approx 0.41$ for $1.3 \mathrm{GeV}$ protons, accelerated in a 2001 April 15 SEP event. Using the Kolmogorov spectrum with $q=5 / 3$ to calculate the dimensionless $\tau \approx 1.089$ from Eq. (18), we would get $\xi^{2} \tau / 3 \approx 0.061$.

The fundamental solution of the telegraph equation is characterised by a sharp propagating front and particle pile-up just ahead of the peak of a travelling pulse, followed by an extended wake. These features, ultimately caused by a finite particle speed, should be contrasted with a symmetrical Gaussian shape of the travelling pulse, predicted by the diffusion approximation that has infinite propagation speed. The asymmetry of the density profile about the peak indicates the limitations of the diffusion approximation. The approximation clearly breaks down ahead of the pulse. These features of the two models are illustrated in Fig. 1 which shows the predicted density profiles, based on the fundamental solutions above, for $t=20$ in the adopted dimensionless variables.

For a more detailed modelling of SEP transport, it might be necessary to impose an additional boundary condition at some $z=z_{0}<0$, which physically would correspond to the acceleration site $z=0$ located close to the solar surface. Yet it is clear from the fundamental solution $F(z, t)$ on the infinite interval that relatively few particles would interact with the boundary unless $\xi>0$, which would cause the density pulse to travel in

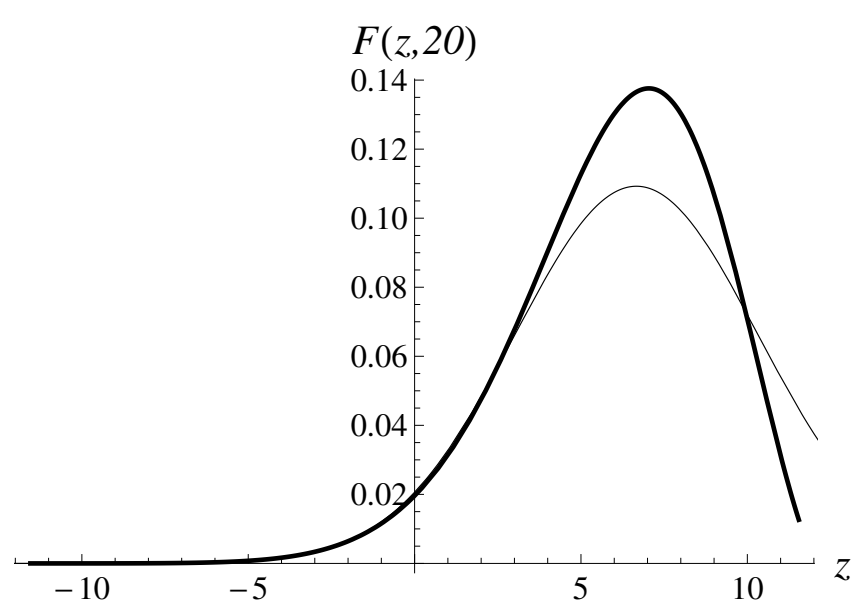

Fig. 1. Predicted travelling pulse solutions in the diffusion approximation (Eq. (21), thin curve) and in the telegraph equation model (Eq. (26), thick curve) for $t=20$. The parameters are $\xi=-1$ and $\tau=1$. The solution to the telegraph equation vanishes for $|z|>t / \sqrt{3 \tau} \approx 11.55$ and, in contrast to that of the diffusion equation, has an asymmetrical shape that leads to shorter rise times for the intensity of solar energetic particles.

the negative $z$-direction. It is also clear that if $\xi<0$, then a reflecting boundary at $z_{0} \approx 0$ would simply lead to doubling the density $F(z, t)$ for all $z>0$. Solutions of the telegraph equation for more general initial and boundary conditions can be obtained by standard methods (e.g., Kevorkian 2000). Since we are primarily interested in comparing our results with those of the diffusion model of Schlickeiser \& Shalchi (2008), we neglect possible boundary effects that could alter the shape of the density pulse.

Now, as in the diffusion limit, the time $t_{\mathrm{m}}$ for $F(z, t)$ to reach a maximum follows from $\mathrm{d} F / \mathrm{d} t=0$, which yields the following implicit equation for $t_{\mathrm{m}}$ :

$\sqrt{\frac{1-\xi^{2} \tau / 3}{1-3 z^{2} \tau / t_{\mathrm{m}}^{2}}}=\frac{I_{0}\left(s_{\mathrm{m}}\right)}{I_{1}\left(s_{\mathrm{m}}\right)}$,

where $s_{\mathrm{m}}$ is the value of $s$ based on $t=t_{\mathrm{m}}$. The asymmetrical shape of the travelling pulse, predicted by the solution to the telegraph equation, should lead to shorter rise times. This is easy to confirm in the limit $\xi \rightarrow 0$, when an asymptotic expansion $I_{\alpha}(s) \approx \sqrt{2 \pi s} \exp (s)\left[1+\left(1-4 \alpha^{2}\right) / 8 s\right]$ of the modified Bessel functions $I_{0}$ and $I_{1}$ in Eq. (29) yields after some algebra

$t_{\mathrm{m}} \approx \frac{3}{2} z^{2}-\tau$

which gives, for example, $t_{\mathrm{m}} \approx 23$ in the case $\xi=0, \tau=1$, $z=4$. This compares well with the numerically computed value of $t_{\mathrm{m}} \approx 22.84$.

More generally, the form of Eq. (29) suggests that, given an observed rise time and a physically motivated pitch-angle scattering rate, the dimensionless parameter $z^{2} \tau / t_{\mathrm{m}}^{2}$ could serve as a convenient empirical measure of the accuracy of the diffusion approximation. Indeed, the condition

$\frac{z^{2} \tau}{t_{\mathrm{m}}^{2}}<1$

is consistent, within a factor of order unity, with both Eq. (30) in the limit $|\xi| z \ll 1$ and Eq. (28) in the limit $|\xi| z \gg 1$, implying that corrections to the predictions of the diffusion approximation should be small as long as Eq. (31) is satisfied. 
Finally, the telegraph equation can be used to derive a modified expression for the streaming anisotropy, valid at short times after injection. Using Eq. (13) in Eq. (23) leads to the following generalisation of Eq. (24):

$A(z, t)=\frac{1}{F}\left(\tau \frac{\partial^{2} F}{\partial t \partial z}-\frac{\partial F}{\partial z}\right)$

According to the fundamental solution given by Eq. (26), the particles do not arrive at a given distance $z$ until the time $t=$ $\sqrt{3 \tau}|z|$, and so the anisotropy remains finite at all times.

\section{Summary}

The Fokker-Planck equation for the evolution of a cosmic-ray distribution function can be simplified to a diffusion equation for the particle density when the particle pitch-angle distribution is weakly anisotropic, in which case the scale of density variation exceeds the scattering length (Jokipii 1966; Hasselmann \& Wibberenz 1970). An important physical effect due to spatial variations of the mean magnetic field is adiabatic focusing that causes coherent particle streaming along the mean field (Earl 1976; Kunstmann 1979). Schlickeiser \& Shalchi (2008) gave a systematic derivation of the diffusion approximation in a weak focusing limit, when the adiabatic focusing length is much greater than the turbulent scattering length of cosmic-ray particles.

The diffusion approximation is the standard approximation for studying the cosmic-ray transport (e.g., Schlickeiser et al. 2009; Artmann et al. 2011; Tautz et al. 2012, and references therein). Hence it is useful to know its limitations that are related to the fact that the signal propagation speed is infinite in the diffusion limit. An improved description of the cosmic-ray transport is provided by the telegraph equation, characterised by a finite propagation speed. In the past, the telegraph equation for focused particle transport was obtained from an eigenfunction expansion of the distribution function, which made it difficult to relate the coefficients of the eigenfunction expansion to the particle density that is the physical quantity of interest (Earl 1976). An alternative derivation of the telegraph equation for the particle density in the case of an arbitrary focusing strength has been recently presented (Litvinenko \& Noble 2013), but it is only valid under the assumption of isotropic pitch-angle scattering.

In this paper we derived the telegraph equation for the particle density in a weak focusing limit. The approach is an extension of the perturbation analysis of Beeck \& Wibberenz (1986). The simplifying assumptions of the analytical treatment are a constant focusing length and the neglect of momentum diffusion, but the derivation is valid for anisotropic pitch-angle scattering, thus complementing the derivation given by Litvinenko \& Noble (2013). It is worth stressing that, while the telegraph equation formally reduces to the diffusion equation when the parameter $\tau \rightarrow 0$, in reality the parameter is of order unity throughout the physically relevant range of the Fokker-Planck pitch-angle scattering rates, calculated using quasilinear theory.

We used the new focused transport equation to re-examine the diffusive propagation of SEPs in interplanetary space.
Specifically, we calculated the fundamental solution of the telegraph equation, determined the time it takes for the particle intensity to reach a maximum at a given distance from the Sun, and compared the results with those obtained by Schlickeiser \& Shalchi (2008) in the diffusion approximation.

From our side-by-side comparison of the predicted evolution of a propagating pulse of energetic particles, we conclude that the predictions of the model based on the telegraph equation are both qualitatively and quantitatively different from those obtained in the diffusion approximation. The telegraph equation predicts an asymmetrical shape of the travelling particle pulse, with a sharp propagating front and an extended wake. A related result is a shorter rise time. We used the analytical solutions to suggest an empirical dimensionless parameter that could be used to quantify the accuracy of the diffusion approximation.

The potentially significant differences of the two transport models suggest that the higher-order, more accurate telegraph equation should be used in analysis of the SEP data, at least to check the accuracy of the predictions of the simpler focused diffusion model.

Acknowledgements. Y.L.'s visit to Ruhr-Universität Bochum, where this work was completed, was partly supported by the Deutsche Forschungsgemeinschaft through a grant to the DFG Research Unit 1048 "Instabilities, Turbulence and Transport in Cosmic Magnetic Fields". R.S. acknowledges support by the Deutsche Forschungsgemeinschaft through grants Schl 201/21-2 and Schl 201/23-1. We acknowledge the referee whose constructive report was very helpful.

\section{References}

Artmann, S., Schlickeiser, R., Agueda, N., Krucker, S., \& Lin, R. P. 2011, A\&A, 535, A92

Beeck, J., \& Wibberenz, G. 1986, ApJ, 311, 437

Bieber, J. W., Dröge, W., Evenson, P. A., et al. 2002, ApJ, 567, 622

Dröge, W., Kartavykh, Y. Y., Klecker, B., \& Kovaltsov, G. A. 2010, ApJ, 709, 912

Earl, J. A. 1974, ApJ, 193, 231

Earl, J. A. 1976, ApJ, 205, 900

Earl, J. A. 1981, ApJ, 251, 739

Gombosi, T. I., Jokipii, J. R., Kota, J., Lorencz, K., \& Williams, L. L. 1993, ApJ, 403, 377

Haggerty, D. K., \& Roelof, E. C. 2002, ApJ, 579, 841

Hasselmann, K., \& Wibberenz, G. 1968, Z. Geophys., 34, 353

Hasselmann, K., \& Wibberenz, G. 1970, ApJ, 162, 1049

Jokipii, J. R. 1966, ApJ, 146, 480

Kevorkian, J. 2000, Partial Differential Equations: Analytical Solution Techniques (Berlin: Springer)

Kirk, J. G., Schlickeiser, R., \& Schneider, P. 1988, ApJ, 328, 269

Kóta, J., Merenyi, E., Jokipii, J. R., et al. 1982, ApJ, 254, 398

Kunstmann, J. 1979, ApJ, 229, 812

Litvinenko, Y. E. 2012, ApJ, 752, 16

Litvinenko, Y. E., \& Noble, P. L. 2013, ApJ, 765, 31

Meyer, P., Parker, E. N., \& Simpson, J. A. 1956, Phys. Rev., 104, 768

Roelof, E. C. 1969, in Lectures in High Energy Astrophysics, eds. H. Ögelman, \& J. R. Wayland (NASA SP-199; Washington, DC: NASA), 111

Sáiz, A., Ruffolo, D., Bieber, J. W., Evenson, P., \& Pyle, R. 2008, ApJ, 672, 650 Schlickeiser, R. 2011, ApJ, 732, 96

Schlickeiser, R., \& Shalchi, A. 2008, ApJ, 686, 292

Schlickeiser, R., Artmann, S., \& Dröge, W. 2009, Open Plasma Phys. J., 2, 1

Schwadron, N. A., \& Gombosi, T. I. 1994, J. Geophys. Res., 99, 19301

Tautz, R. C., Dosch, A., \& Lerche, I. 2012, A\&A, 545, A149

Webb, G. 1985, ApJ, 296, 319 


\section{Appendix A: Particle transport in a moving medium}

In the solar wind and various other astrophysical situations, cosmic-ray transport often occurs in a moving medium. When this is the case, the Fokker-Planck Eq. (1) holds in a co-moving reference frame, i.e. in the rest frame of a moving plasma. Below we generalise the analysis of the paper by deriving a diffusive transport equation for cosmic-ray particles in a moving background plasma.

Suppose the plasma, supporting electromagnetic fluctuations that interact with the particles, moves with respect to the observer with a bulk speed $\boldsymbol{U}=U \hat{\boldsymbol{z}}$ along the mean magnetic field $\boldsymbol{B}_{0}=B_{0} \hat{z}$. It is convenient to transform to the mixed co-moving coordinate system (Webb 1985; Kirk et al. 1988), in which the time $t$ and position $\boldsymbol{x}$ are measured in the laboratory (observer) coordinate system, whereas the particle momentum $\boldsymbol{p}^{*}$ is measured in the rest frame of the streaming plasma. Equation (1) is written in these variables as follows:

$$
\begin{gathered}
\Gamma\left[1+\frac{U v^{*} \mu^{*}}{c^{2}}\right]\left[\frac{\partial f}{\partial t}-\frac{1}{v^{*}} \frac{\partial U}{\partial t} \Gamma^{2}\left(\mu^{*} p^{*} \frac{\partial f}{\partial p^{*}}+\left(1-\mu^{* 2}\right) \frac{\partial f}{\partial \mu^{*}}\right)\right] \\
+\Gamma\left[U+v^{*} \mu^{*}\right]\left[\frac{\partial f}{\partial z}-\frac{1}{v^{*}} \frac{\partial U}{\partial z} \Gamma^{2}\left(\mu^{*} p^{*} \frac{\partial f}{\partial p^{*}}+\left(1-\mu^{* 2}\right) \frac{\partial f}{\partial \mu^{*}}\right)\right] \\
+\frac{v^{*}\left(1-\mu^{* 2}\right)}{2 L} \frac{\partial f}{\partial \mu^{*}}=\frac{\partial}{\partial \mu^{*}}\left(D_{\mu^{*} \mu^{*}} \frac{\partial f}{\partial \mu^{*}}\right)
\end{gathered}
$$

where $\Gamma=\left[1-\left(U^{2} / c^{2}\right)\right]^{-1 / 2}$ is the bulk Lorentz factor of the flow.

For brevity we drop the $*$-notation in what follows, keeping in mind that the phase-space coordinates are to be taken in the mixed co-moving coordinate system. Moreover, for simplicity we restrict our analysis to spatially varying, but stationary flows, $U=U(z)$, so that Eq. (A.1) becomes

$$
\begin{aligned}
\Gamma[1 & \left.+\frac{U v \mu}{c^{2}}\right] \frac{\partial f}{\partial t} \\
& +\Gamma[U+v \mu]\left[\frac{\partial f}{\partial z}-\frac{1}{v} \frac{\partial U}{\partial z} \Gamma^{2}\left(\mu p \frac{\partial f}{\partial p}+\left(1-\mu^{2}\right) \frac{\partial f}{\partial \mu}\right)\right]+\frac{v \mu f}{L} \\
& =\frac{\partial}{\partial \mu}\left(D_{\mu \mu} \frac{\partial f}{\partial \mu}-\frac{v\left(1-\mu^{2}\right) f}{2 L}\right) .
\end{aligned}
$$

We proceed to generalise the argument of Sect. 2 to the case of a moving background plasma. We substitute Eq. (2) in Eq. (A.2) and use Eq. (3) to obtain

$$
\begin{aligned}
\Gamma[1+ & \left.\frac{U v \mu}{c^{2}}\right] \frac{\partial F}{\partial t} \\
& +\Gamma\left[1+\frac{U v \mu}{c^{2}}\right] \frac{\partial g}{\partial t}+\Gamma[U+v \mu]\left[\frac{\partial F}{\partial z}-\frac{1}{v} \frac{\partial U}{\partial z} \Gamma^{2} \mu p \frac{\partial F}{\partial p}\right] \\
& +\Gamma[U+v \mu]\left[\frac{\partial g}{\partial z}-\frac{1}{v} \frac{\partial U}{\partial z} \Gamma^{2}\left(\mu p \frac{\partial g}{\partial p}+\left(1-\mu^{2}\right) \frac{\partial g}{\partial \mu}\right)\right]+\frac{v \mu g}{L} \\
= & \frac{\partial}{\partial \mu}\left(D_{\mu \mu} \frac{\partial g}{\partial \mu}-\frac{v\left(1-\mu^{2}\right) g}{2 L}\right) .
\end{aligned}
$$

Now averaging over $\mu$ yields

$$
\begin{aligned}
\Gamma\left[\frac{\partial F}{\partial t}\right. & \left.+U \frac{\partial F}{\partial z}\right]-\frac{\Gamma^{3}}{3} \frac{\partial U}{\partial z} p \frac{\partial F}{\partial p} \\
& +\frac{\Gamma v}{2}\left[\frac{U}{c^{2}} \frac{\partial}{\partial t}+\frac{\partial}{\partial z}+\frac{1}{\Gamma L}-\frac{\Gamma^{2}}{2 v^{2}} \frac{\partial U^{2}}{\partial z}\left(p \frac{\partial}{\partial p}+2\right)\right] \int_{-1}^{1} \mathrm{~d} \mu \mu g \\
& -\frac{\Gamma^{3}}{2} \frac{\partial U}{\partial z}\left[p \frac{\partial}{\partial p}+3\right] \int_{-1}^{1} \mathrm{~d} \mu \mu^{2} g=0 .
\end{aligned}
$$

Next we subtract Eq. (A.4) from Eq. (A.2) and express the anisotropy $g(\mu)$ in terms of the isotropic distribution function $F$, assuming that the spatial gradients of the plasma flow velocity are small. The resulting approximate equation for $g$ is as follows:

$$
\Gamma \nu \mu\left[\frac{U}{c^{2}} \frac{\partial F}{\partial t}+\frac{\partial F}{\partial z}+\frac{\partial g}{\partial z}\right]+\Gamma \frac{\partial g}{\partial t}=\frac{\partial}{\partial \mu}\left(D_{\mu \mu} \frac{\partial g}{\partial \mu}-\frac{v\left(1-\mu^{2}\right) g}{2 L}\right) .
$$

As in the analysis of Sect. 2 , in the weak focusing limit, $\lambda_{0} / L \ll$ 1 , this equation can be approximately integrated to give

$$
\frac{\partial g}{\partial \mu}=-\frac{\Gamma v\left(1-\mu^{2}\right)}{2 D_{\mu \mu}(\mu)}\left[\frac{U}{c^{2}} \frac{\partial F}{\partial t}+\frac{\partial F}{\partial z}\right]
$$

where we used $D_{\mu \mu}( \pm 1)=0$. Now integrating Eq. (A.6) yields

$g(\mu)=c_{0}-\frac{\Gamma v}{2}\left[\frac{U}{c^{2}} \frac{\partial F}{\partial t}+\frac{\partial F}{\partial z}\right] \int_{-1}^{\mu} \mathrm{d} x \frac{\left(1-x^{2}\right)}{D_{\mu \mu}(x)}$.

On using Eq. (3) to determine the integration constant $c_{0}$, we obtain the following generalisation of Eq. (7):

$$
\begin{aligned}
g(\mu)= & \frac{\Gamma v}{4}\left[\frac{U}{c^{2}} \frac{\partial F}{\partial t}+\frac{\partial F}{\partial z}\right] \\
& \times\left[\int_{-1}^{1} \mathrm{~d} \mu \frac{(1-\mu)\left(1-\mu^{2}\right)}{D_{\mu \mu}(\mu)}-2 \int_{-1}^{\mu} \mathrm{d} x \frac{\left(1-x^{2}\right)}{D_{\mu \mu}(x)}\right] .
\end{aligned}
$$

To complete the derivation of the diffusive approximation for particle transport in a moving plasma, we integrate Eq. (A.8) to determine the two integrals that appear in Eq. (A.4):

$$
\begin{aligned}
\int_{-1}^{1} \mathrm{~d} \mu \mu g(\mu) & =-\frac{\Gamma v}{4}\left[\frac{U}{c^{2}} \frac{\partial F}{\partial t}+\frac{\partial F}{\partial z}\right] \int_{-1}^{1} \mathrm{~d} \mu \frac{\left(1-\mu^{2}\right)^{2}}{D_{\mu \mu}(\mu)} \\
& =-\frac{2 \Gamma \kappa_{\|}}{v}\left[\frac{U}{c^{2}} \frac{\partial F}{\partial t}+\frac{\partial F}{\partial z}\right]
\end{aligned}
$$

where we used Eqs. (9) and (10), and

$$
\begin{aligned}
& \int_{-1}^{1} \mathrm{~d} \mu \mu^{2} g(\mu)=-\frac{\Gamma v}{6}\left[\frac{U}{c^{2}} \frac{\partial F}{\partial t}+\frac{\partial F}{\partial z}\right] \\
& \times\left[\int_{-1}^{1} \mathrm{~d} \mu \frac{(1-\mu)\left(1-\mu^{2}\right)}{D_{\mu \mu}(\mu)}-\int_{-1}^{1} \mathrm{~d} \mu \frac{\left(1-\mu^{3}\right)\left(1-\mu^{2}\right)}{D_{\mu \mu}(\mu)}\right] \\
& =\frac{\Gamma v}{6}\left[\frac{U}{c^{2}} \frac{\partial F}{\partial t}+\frac{\partial F}{\partial z}\right] \int_{-1}^{1} \mathrm{~d} \mu \frac{\mu\left(1-\mu^{2}\right)^{2}}{D_{\mu \mu}(\mu)}
\end{aligned}
$$

Recall that the scattering coefficient is an even function, $D_{\mu \mu}(-\mu)=D_{\mu \mu}(\mu)$, when scattering is isotropic, $D_{\mu \mu}(\mu)=$ $D_{0}\left(1-\mu^{2}\right)$, or more generally when the coefficient is calculated using quasilinear theory (Eq. (16)). Hence the function $g(\mu)$ is odd, and so the second moment given by Eq. (A.10) vanishes. On substituting the first moment (A.9) into Eq. (A.4) and rearranging terms, we find that the isotropic part of the cosmicray phase-space distribution in the weak adiabatic focusing limit obeys the following diffusion-convection equation:

$$
\begin{aligned}
{[1} & \left.+\frac{2 \Gamma^{3} U^{2} \partial_{z} U \kappa_{\|}}{v^{2} c^{2}}-\frac{U \kappa_{\|}}{L c^{2}}\right] \frac{\partial F}{\partial t}+\left[U-\frac{\kappa_{\|}}{L}+\frac{2 \Gamma^{3} U \partial_{z} U \kappa_{\|}}{v^{2}}\right] \frac{\partial F}{\partial z} \\
- & \frac{\Gamma^{2}}{3} \frac{\partial U}{\partial z} p \frac{\partial F}{\partial p}-\frac{\Gamma U^{2} \kappa_{\|}}{c^{4}} \frac{\partial^{2} F}{\partial t^{2}}-\Gamma \kappa_{\|} \frac{\partial^{2} F}{\partial z^{2}} \\
& -\frac{2 \Gamma U \kappa_{\|}}{c^{2}} \frac{\partial^{2} F}{\partial t \partial z}+\frac{\Gamma^{3} U \partial_{z} U \kappa_{\|} p}{v^{2}}\left[\frac{U}{c^{2}} \frac{\partial^{2} F}{\partial t \partial p}+\frac{\partial^{2} F}{\partial z \partial p}\right]=0,
\end{aligned}
$$

which reduces to Eq. (11) if $U=0$. 
We conclude that the bulk flow of a background plasma leads to a modified telegraph equation for the cosmic-ray density already in the diffusion approximation. The equation above should be used for relativistic flows, such as those present in gammaray burst sources and jets of active galactic nuclei. For the
SEP transport in the solar wind, though, the main corrections to the diffusive transport model are given by the convective term $U \partial_{z} F$ and the adiabatic energy loss term $\Gamma^{2}\left(\partial_{z} U\right) p \partial_{p} F / 3$. We neglect the latter term throughout the paper, assuming that $\partial_{z} U$ is small enough. 